\title{
The Natural Way of Gestures for Interacting with Smart TV
}

\author{
Jinhae Choi, Jiyoung Hong
}

UX Lab., LG Electronics Inc., Seoul, 137-130

\begin{abstract}
Objective: The aim of this study is to get an optimal mental model by investigating user's natural behavior for controlling smart TV by mid-air gestures and to identify which factor is most important for controlling behavior. Background: A lot of TV companies are trying to find simple controlling method for complex smart TV. Although plenty of gesture studies proposing they could get possible alternatives to resolve this pain-point, however, there is no fitted gesture work for smart TV market. So it is needed to find optimal gestures for it. Method: (1) Eliciting core control scene by in-house study. (2) Observe and analyse 20 users' natural behavior as types of hand-held devices and control scene. We also made taxonomies for gestures. Results: Users' are trying to do more manipulative gestures than symbolic gestures when they try to continuous control. Conclusion: The most natural way to control smart TV on the remote with gestures is give user a mental model grabbing and manipulating virtual objects in the mid-air. Application: The results of this work might help to make gesture interaction guidelines for smart TV.
\end{abstract}

Keywords: Smart TV, Gesture interaction, Natural interaction, Remote controller, Pantomimic gesture

\section{Introduction}

최근 활발히 보급되고 있는 스마트 TV는 전통적 TV와 달리 수백 종 이상의 많은 컨텐츠를 제공할 뿐 아니라 게임 /교육 등 능동적인 참여를 요구하는 컨텐츠까지 제공되고 있 다. 이에 따라 필연적으로 복잡성이 증가되고 사용자에게 능동적인 참여가 요구된다. 또한 스마트 TV의 주된 사용 방식은 컨텐츠 소비에 있어서도 전통적인 채널 Up/Down 뿐 아니라 텍스트 검색, 연관 검색 등 $\mathrm{PC}$ 에서 활용하던 검 색 행태로 옮아가고 있을 뿐 아니라, 다양한 Application, Web Browsing, 실시간 SNS 등 보다 폭넓고 풍부한 기능 들을 제공하는 스마트 TV가 보편화되고 있는 상황이다.

그러나 스마트 TV 컨트롤에는 아직 통일된 양식이나 관 습(convention)이 존재하지 않아서 제조사 별로, 심지어는
동일 제조사의 각 모델 별로 상이한 과업(Task) 수행 방식 을 학습해야만 한다. 뿐만 아니라 스마트폰, 타블렛 $\mathrm{PC}$, 기 타 액세서리를 $\mathrm{TV}$ 조작에 활용할 수 있는 환경이 조성됨에 따라 하나의 기기와 인터랙션하는 방식이 어떤 입력장치 (hand-held input device)를 이용하느냐에 따라 천차만별 인 상황이다.

리모컨의 형태만으로 보아도, 버튼 누름 위주의 기존 리 모컨 뿐 아니라, L사의 모션 리모컨, S사의 터치패드형 리 모컨, A사의 심플한 4방향 방식 리모컨. G사의 QWERTY 와 조이스틱 형태의 리모컨 등 다양한 형태와 조작 방법의 리모컨을 제공하고, 게다가 스마트폰(Smartphone), 태블릿 (Tablet) 등의 다양한 Handheld Device들과의 무선 연결 을 통해 App 형태로 리모컨 기능이 수행 가능하다. 이처럼 $\mathrm{TV}$ 제조사들은 다양한 리모컨과 혁신적인 UI로 사용자 경 험(User Experience)의 개선을 꾀하고 있다(Figure 1). 이

Corresponding Author: Jinhae Choi. UX Lab., LG Electronics Inc., Seoul, 137-130.

Phone: +82-2-8220-2000, E-mail: jin.choi@lge.com

Copyright@2012 by Ergonomics Society of Korea(pISSN:1229-1684 eISSN:2093-8462). All right reserved.

(c) This is an open-access article distributed under the terms of the Creative Commons Attribution Non-Commercial License(http://creativecommons.org/licenses/by-nc/3.0/), which permits unrestricted non-commercial use, distribution, and reproduction in any medium, provided the original work is properly cited. http://www.esk.or.kr 
와 같은 비정형적인 다양화는 제품 주기의 초기 단계에서 흔 히 보이는 현상으로, 일부 Early Adopter들에게는 반가운 현상일 수 있으나 오히려 대부분의 일반적인 사용자에게는 큰 혼란을 가져오고 제조사가 애써 제공한 많은 기능과 컨 텐츠들을 충분히 즐기지 못하게 하는 요인이 되고 있음이 명백해 보인다.

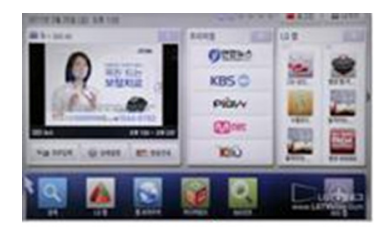

A사

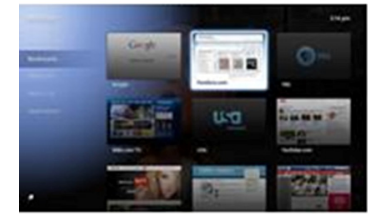

사
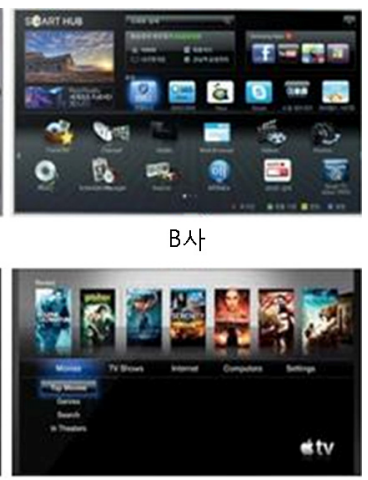

$\mathrm{D}$ 사

Figure 1. Diversity of user interface of Smart TV

또한 사용자 행동 인식(Behavior recognition) 기술 기반 으로 제스쳐(Gesture) 및 음성(Voice) 컨트롤 기능을 지원 하는 모델도 있으나 매우 제한적이고, 인식률이 극히 낮으며, 실제 사용 행태를 관찰해 보면 약간의 흥미를 끌 뿐 지속적 인 사용이 이루어지고 있지 않음을 볼 때, 정말 사용자 입 장에서 필요한 기능을 제공하고 있는 것인지 의심스럽기도 하다.

이러한 결과는 결국 어떠한 기능이나 인식 기술을 제공하 느냐 뿐 아니라 어떠한 Scene에서 어떻게 제공해야 하느냐, 즉 총체적인 사용자 경험을 만족시키기 위한 세심한 노력이 부족했기 때문으로, 깊이 있는 연구가 없는 단순한 기술 자 랑식의 제품 설계는 오히려 사용자에게 고통만을 배가해줄 따름이다.

Smart TV의 최적 사용자 경험을 찾기 위해서는 근본적으 로 현재의 Smart TV 경험이란 어떠한 것인지에 대한 사용 자 멘탈 모델을 확인하고, 사용성(Usabilty)을 최적화 할 수 있는 컨트롤 방법 및 디바이스에 대한 근본적(fundamental) 인 연구가 절실하다.

따라서 본 연구에서는 Smart TV의 주요 사용 Activity 로부터 핵심 가치(Key value)를 추출하고, 핵심 조작 상황 (Core control scene) 을 도출하였다. 단순히 현재의 리모 컨에 있는 명령어가 아니라 핵심 조작 상황을 포괄하는 Command 세트를 정의하고 Command로 TV를 컨트롤하는 최적의 방법을 알아내기 위해 실험을 통해 사용자들이 진정
으로 원하는 조작 방식을 탐색적 연구를 통해 알아보고자 한다.

\section{Method}

\subsection{Core control scene}

스마트 TV를 사용함에 있어 사용자가 가장 많이 사용하 면서 중요하게 생각하고 있는, 이른바 사용자의 사용 접점빈 도가 가장 높은 Scene을 Core Control Scene으로 명명하 였다. 이 Scene들을 도출하기 위하여 숙련자(Expert) 및 초심자(Novice User)를 대상으로 심층 인터뷰를 진행하였 고 A사 Smart TV 사용자를 대상으로 한 체험담 리뷰를 분 석하여 도출하였다.

\subsubsection{User depth interview}

Smart TV 사용자 패널 5명 (대학생 남자 3, 여자 2)을 대 상으로 사용자의 일반적인 Daily Routine을 바탕으로 가족 구성원과 가정 내 전체적 정황을 파악하기 위해 오전, 오후, 저녁시간 및 평일, 휴일을 구분하여 사용자의 TV 사용 환경 과 습관에 관하여 인터뷰를 실시하였다. 또한 과제로서 평일, 휴일 동안에 개인적인 $\mathrm{TV}$ 사용 목적 및 목적에 따른 행동 Flow를 기록하는 목적카드를 기록하게 하였으며 하루를 2 시간 단위로 기록하는 User Diary 및 TV 사용 환경 Scene 을 작성하게 하였다(Figure 2). 그 결과 사용자 TV 사용 환 경 Scene은 Setting-up, Choosing, Consuming, Managing, Sharing 등의 5 가지로 사용 행동들이 분류 및 정리가 되 었다.

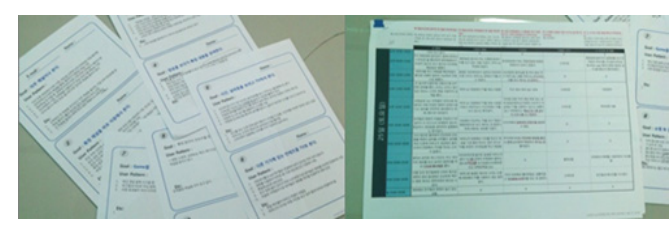

Figure 2. Goal and behavior flow card

\subsubsection{Major activities for using smart TV}

Smart TV 체험단 및 Early adopter 블로그 리뷰를 바탕 으로 TV 경험의 Activity를 추출하였으며 이를 사용자 심층 인터뷰를 통해 도출된 5가지의 Scene을 기준으로 Figure 3 와 같은 Scene별 Activity를 추출할 수 있었다. 이 Activity 들과 인터뷰 결과에서 나온 주요 Painpoint들을 Affinity diagram 방식으로 분석하여 그룹화를 반복한 결과 크게 '쉽 
고 편하게(Easy of use)', '바로바로 빠르게(Quick Access)', '편안한 Web 사용(Comfortable Web)', '3D 경험(3D Experience)'이라는 4 가지 핵심 가치를 도출하였다.

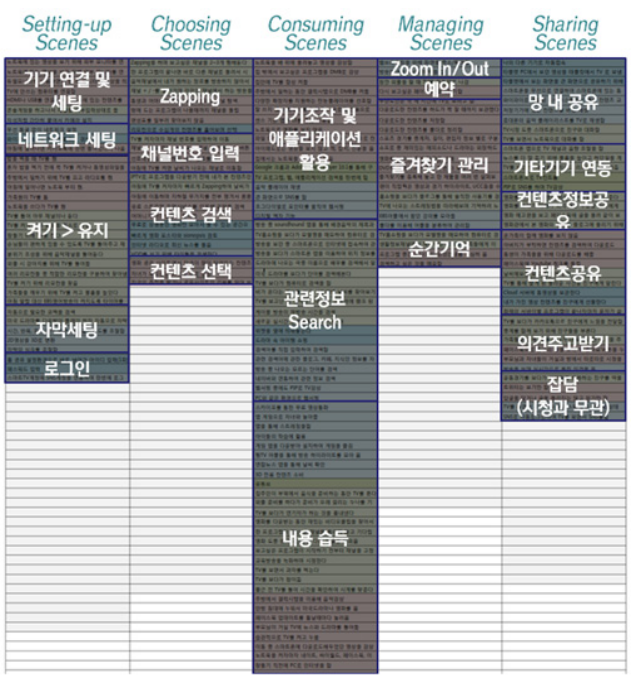

Figure 3. Activity analysis of smart TV usage scene

이 핵심 가치가 추출된 Interview 내용을 역추적하여 근 본적인 Needs가 발굴된 사용 scene을 모두 수집한 결과 총 13개의 Core Control Scene인 것으로 나타났다(Figure 4). 이 Scene들은 실험에서 기준 Command set을 정하는 기준으로 사용되었다.

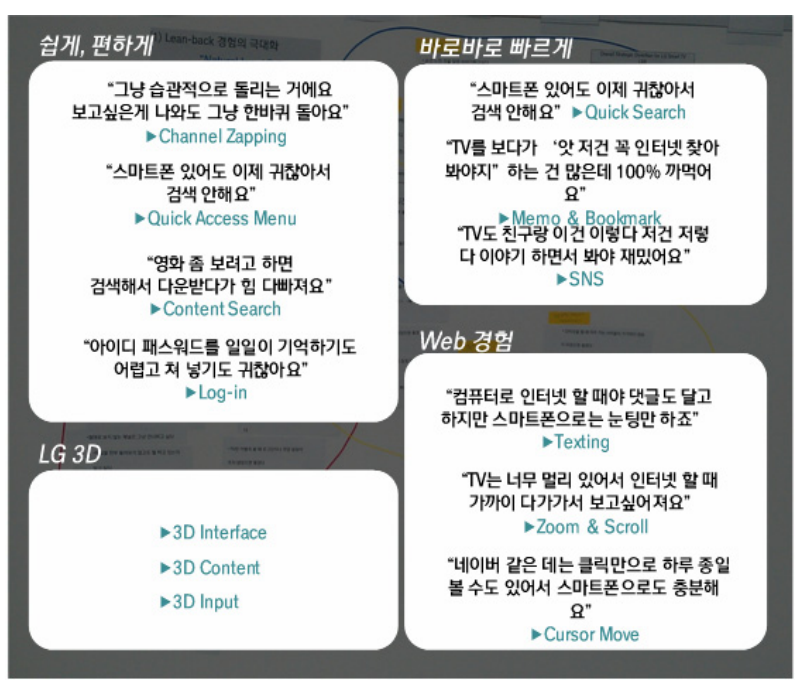

Figure 4. Key value and core control scenes
그 결과 Smart TV의 Core Control Scene은 Lean-back 경험의 극대화를 위한 Channel Zapping, Quick Access Menu, Content Search, Log-in 등 4개 Scene, Live 시 청 및 풍부한 정보제공을 위한 Quick Search, Memo \& Bookmark, SNS 등 3개 Scene, 직관적인 Web browsing 경험을 위한 Texting, Zoom \& Scroll, Cursor Move 등 3 개 Scene, $3 \mathrm{D}$ 기능 사용시 동일한 사용 경험을 위한 $3 \mathrm{D}$ Interface, 3D Content, 3D Input 등 3개 Scene 등 총 13 개 Scene으로 정의가 되었다.

\subsection{Gesture interaction studies}

사용자의 다양한 Gesture를 유형화하기 위해 행위 Interaction에 관한 문헌 탐색조사를 하였다. 그 결과 문헌 에서 정의된 행위들이 크게 4 가지(순간적으로 일어나는 특 정 동작에 적합한 행동, 대상물을 실제 있는 것처럼 연속적 으로 조작하는 행동, 특정 위치를 가리키는 행동, 언어를 대 신하는 행동)로 분류가 되었다(Figure 4). 이를 바탕으로 제스쳐 방식 기준으로 재 분류한 결과 4 가지 대분류 안에 서 7 가지의 제스쳐 방식에 대한 기준을 정립할 수 있었다 (Buxton, 2011; Karam, 2005; Kendon, 1988).

두 번째로 기존 제스쳐 연구를 참조하여 스마트 $\mathrm{TV}$ Operation을 위한 Command 리스트를 확보하기 위해 Core Control Scene을 바탕으로 문헌 탐색조사를 하였다. 그 결과 Table 1과 같이 전원, 취소, 메뉴실행, 순간저장, 볼륨, 스크 롤, 채널, 줌, 지정, 입력 등의 10가지 Operation Command

Table 1. Taxonomies of gestures

\begin{tabular}{c|c|c|c}
\hline \multirow{4}{*}{$\begin{array}{c}\text { Buxton, B. } \\
\text { (2011) }\end{array}$} & Symbolic & "특정 동작" & Symbolic \\
\cline { 2 - 3 } & Iconic & "가리키기" & Pointing \\
\cline { 2 - 3 } & Pantomimic & "시늉하기" & Manipulative \\
\hline \multirow{4}{*}{$\begin{array}{c}\text { Karam, M } \\
\text { (2005) }\end{array}$} & Gesticulation & "Speech 보조" & \\
\cline { 2 - 4 } & Manipulative & "조작하기" & Manipulative \\
\cline { 2 - 4 } & Deictic & "가리키기" & Pointing \\
\cline { 2 - 4 } & Semaphoric & "특정 동작" & Symbolic \\
\cline { 2 - 4 } & Sign language & "수화" & Language \\
\hline \multirow{4}{*}{$\begin{array}{c}\text { Kendon } \\
(1988)\end{array}$} & Gesticulation & "Speech 보조" & \\
\cline { 2 - 4 } & Language like & "언어유사" & Language \\
\cline { 2 - 4 } & Pantomimes & "시늉하기" & Manipulative \\
\cline { 2 - 4 } & Emblems & "특정 동작" & Symbolic \\
\cline { 2 - 3 } & Sign language & "수화" & Language \\
\hline
\end{tabular}


를 도출하였다(Lee, 2010; Holman, 2005; Wobbrock, 2009; Neßelrath, 2011).

도출된 Core Control Scene과 기존 제스쳐 분류 연구들 은 사용자가 TV를 사용할 때 활용할 수 있는 Natural 제 스쳐를 수집하여 분석하는 기준으로 활용되었다.

\subsection{Experiment}

본 연구에서는 TV 사용자들이 아무런 제약이 존재하지 않을 때 어떠한 방식으로 조작하기를 원하는지 알아내고자 하였다. 기존 TV 리모컨은 모든 동작이 버튼 프레스 방식으 로 구현되어 있으나 이러한 제약이 없고 다양한 명령을 내려 야 한다면 새롭고 다양한 형태의 제스쳐가 도출될 가능성이 높다.

따라서 제약조건이 없이 사용자에게 명령 내용을 제시하 고 이에 적합한 자유로운 모션을 취하도록 하여 이를 유형 별로 분석하여 고빈도로 나타나는 조작을 찾아내고자 하였 다. 단, 리모컨의 형태에 따라 조작 방식이 영향을 받을 수 있어, 현존하는 각종 리모컨 형태를 조건으로 고려하여 실험 을 진행하였다.

Table 2. Major control command of displays

\begin{tabular}{|c|c|c|}
\hline \multirow{4}{*}{$\begin{array}{l}\text { Lee, S. } \\
(2010)\end{array}$} & On / Off & Next / Previous \\
\hline & Open / Close & Scrap \\
\hline & Delete & Copy to other device \\
\hline & Zoom-in / Out & \\
\hline \multirow{5}{*}{$\begin{array}{l}\text { Holman, D. } \\
\text { (2005) }\end{array}$} & Activate & Views \\
\hline & Select & Annotate \\
\hline & Copy \& Paste & Size \\
\hline & Scroll & Share \\
\hline & Browse & Open, Save \& Close \\
\hline \multirow{7}{*}{$\begin{array}{c}\text { Wobbrock, J.O. } \\
\text { (2009) }\end{array}$} & Move / Rotate & Accept / Reject \\
\hline & Duplicate / Paste & Menu access \\
\hline & Previous / Next & Open / Close \\
\hline & Select & Enlarge \\
\hline & Maximize / Minimize & Insert \\
\hline & Cut / Delete & Undo \\
\hline & Zoom-in / out & Shrink \\
\hline \multirow{3}{*}{$\begin{array}{l}\text { Neßelrath, R. } \\
\text { (2011) }\end{array}$} & On / Off & Channel next \\
\hline & Volume increase & Channel previous \\
\hline & Volume decrease & Toggle mute \\
\hline
\end{tabular}

\subsubsection{Participants}

실험대상자는 일반인 참여자를 대상으로 $1: 1$ 개별 인터뷰 형식으로 진행을 하였으며 다양한 제스처가 충분히 수집될 수 있도록 상대적으로 표현력이 좋은 젊은 층을 대상으로 하였다(Table 3).

사전 연구(Cho et al., 2012)에서와 마찬가지로 스마트 $\mathrm{TV}$ 사용 경험이 없는 다양한 전공의 일반 20대 남녀 20 명을 유사 비율로 모집하여 $1: 1$ 심층 인터뷰를 진행하였다. 이 중 스마트폰 사용 유경험자는 $98 \%$, Nintendo Wii나 Microsoft Kinnect 등과 같이 멀티 센서를 이용한 게임의 유경험자는 $48 \%$ 였다.

Table 3. Key value and core control scenes

\begin{tabular}{c|c|c}
\hline \multicolumn{2}{c|}{ 구분 } & Detail \\
\hline \multicolumn{2}{c}{ Number of participants } & 20 \\
\hline \multirow{2}{*}{ Demographics } & Gender & Male :Female =1:1 \\
\cline { 2 - 3 } & Age & 20's (20 29) \\
\hline \multicolumn{2}{c}{ Survey method } & Individual interview \\
\hline
\end{tabular}

\begin{tabular}{|c|l|}
\hline 준비 & $\begin{array}{l}\text { - 과제 및 가상 대상물 설명 } \\
\text { - 참여자 순서로 task를 Balancing함 }\end{array}$ \\
\hline 리모컨 평가 & $\begin{array}{l}\text { - 리모컨을 사용하면서 Operation Command를 } \\
\text { 제스처로 표현 }\end{array}$ \\
\hline 사후설문 & - 인구통계학적 지표 및 제스처 관련 기기 사용 경험 확인 \\
\hline 마무리 & $\begin{array}{l}\text { - 기타 사항 및 Needs 확인 } \\
\text { - 마무리 인사 }\end{array}$ \\
\hline
\end{tabular}

Figure 5. Experiment procedure

\subsubsection{Major control commands}

다수의 사용자가 불특정 대상에 대하여 보편적으로 취하 는 조작 행동 방식을 탐색하기 위하여, TV 스크린만이 아닌 자유롭게 변화/변태/이동하는 임의의 대상을 떠올리도록 하 였고, 기존의 사용 경험을 넘어 다양한 인터랙션이 가능한 입력장치를 상상하도록 모호화(ambiguation) 하였다.

조작 대상이 TV임을 유추하도록 하는 단어가 포함된 경 우나 기존 전자제품에서의 경험이 강하게 반영될만한 명령 어는 인터랙션의 목적을 그대로 반영하여 명령어를 재정의 하였다(Table 4). 예를 들면, '채널', '볼륨' 등은 '다음/이전 단계로', '더 크게/작게 들리도록'과 같이, '전원 켜기/끄기', '스 크롤 하기' 등은 '조작 가능/불가능한 상태로', '상하좌우로 
이동'과 같이 조정하였다. 스마트 $\mathrm{TV}$ 조작을 위한 새로운 필 수 명령으로서 포함된 '메뉴', '홈으로' 등은 인터랙션이 완료 되고 난 후의 모습(scene)을 제공하여 그 중간 과정을 자연 스럽게 떠올리도록 하였다.

\subsubsection{Input devices}

또한 입력장치는 Table 4 와 같이, 맨손(bare-hand) 상황 을 포함하여 패드 (pad)형, 바(bar)형, 스틱(stick)형의 네 가지로 다양화 하였고, 최근 보편적으로 사용되고 있는 스마 트 기기의 크기를 반영하되 버튼 및 특정 제품의 아이덴티티 를 보이는 특징을 제거하여 더미(dummy) 형태로 제작하 였다.

Table 4. Abstracted command definition

\begin{tabular}{|c|c|c|}
\hline \multicolumn{2}{|c|}{ 기본 명령어 } & 재 정의된 기본 명령어 \\
\hline \multirow{2}{*}{ Power } & 전원조작_켜기 & $\begin{array}{l}\text { 대상을 조작 가능한 상태로 } \\
\text { 만드시오 }\end{array}$ \\
\hline & 전원조작_끄기 & $\begin{array}{l}\text { 대상을 조작 불가능한 상태로 } \\
\text { 만드시오 }\end{array}$ \\
\hline Cancel & 취소 & 방금 한 행동을 취소하시오 \\
\hline \multirow{2}{*}{ Menu } & 메뉴 & 선택할 수 있는 것을 불러내시오 \\
\hline & 홈으로 & 숨겨둔 기능이 실행되도록 하시오 \\
\hline Capture & 캡쳐 & 현재상태를 저장하시오 \\
\hline \multirow{2}{*}{ Volume } & 볼륨 조절_+ & 더 크게 들리도록 하시오 \\
\hline & 볼륨 조절_- & 더 작게 들리드록 하시오 \\
\hline Scroll & 스크롤 & 상하좌우로 이동하시오 \\
\hline \multirow{2}{*}{ Channel } & 채널 변경_+ & 다음 단계로 이동하시오 \\
\hline & 채널 변경_- & 이전 단계로 이동하시오 \\
\hline \multirow{2}{*}{ Zoom } & 줌_+ & 더 크게 보이도록 하시오 \\
\hline & 줌_- & 더 작게 보이도록 하시오 \\
\hline Select & 화면요소 선택 & 여러 가지 중 하나를 선택하시오 \\
\hline \multirow{2}{*}{ Input } & 도형 입력 & 동그라미를 그리시오 \\
\hline & 텍스트 입력 & 본인의 이름을 입력하시오 \\
\hline
\end{tabular}

현재 Operation Command를 수행 시 스마트 TV에서는 Point \& Click 또는 버튼 Press 방식으로 제공되고 있다. 본 실험에서는 스마트 TV에서 Operation Command 수행 시 사용자 본연의 직관을 만족시킬 수 있는 Interaction을 탐색 하기 위해 양산 모델 스마트 TV 대상(A사)으로 실험을 수 행하였다. 또한 리모컨의 버튼은 실험 결과에 영향을 줄 수 있는 요인이기 때문에 버튼이 없는 Dummy Mock-up으로 Table 5의 순서대로 실험을 수행하였다.
Table 5. Types of input devices

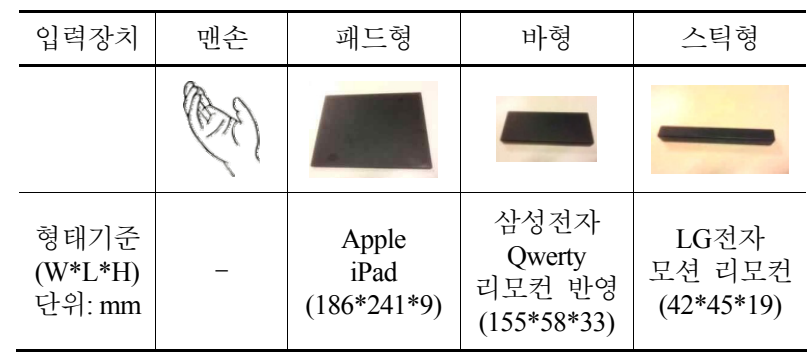

\subsection{Result}

실험 결과를 종합/분석한 결과, 조작 행동 방식을 크게 두 개의 기준으로 유형화 할 수 있었다. 첫 번째는 표면적으로 보여지는 행동 형태(behavior)에 따른 구분이고, 두 번째는 그 행동의 의도나 목적(intent/objective) 에 따른 구분이다.

사용자의 행동 형태에 따른 분류로 보면, 표면적으로 관찰 가능한 세 가지 형태 유형, 즉 가상버튼이나 실제하는 버튼 을 누르거나 터치하는 행동(button press/touch), 입력장치 의 표면 위에서 일어나는 행동(surface gesture), 그리고 입 력장치를 들고 허공에서 취하는 행동(space gesture)으로 구분할 수 있었다(Table 6).

Table 6. Type of gestural behavior based physical motion

\begin{tabular}{|c|c|c|}
\hline 구분 & 설명 & 예시 \\
\hline $\begin{array}{c}\text { Button } \\
\text { Press/Touch }\end{array}$ & $\begin{array}{c}\text { 입력장치의 표면이나 } \\
\text { 가상의 표면 위에 있는 } \\
\text { 버튼을 물리적으로 } \\
\text { 누르거나 터치함 }\end{array}$ & \\
\hline $\begin{array}{l}\text { Surface } \\
\text { gesture }\end{array}$ & $\begin{array}{c}\text { 입력장치의 표면 위에 } \\
\text { 손가락을 대고 움직임을 } \\
\text { 입력 }\end{array}$ & \\
\hline $\begin{array}{l}\text { Space } \\
\text { gesture }\end{array}$ & $\begin{array}{l}\text { 입력장치를 손에 쥔 } \\
\text { 상태로 공간 안에서 } \\
\text { 신체의 일부를 움직임 }\end{array}$ & \\
\hline
\end{tabular}

\subsubsection{Types of gestures as intention}

Buxton(2011)은 가장 무의식적인 몸짓인 제스티큘레이 션 (gesticulation) 으로부터 수화와 같이 심볼을 사인으로 사 용하는 경우(symbolic gesture)에 이르기 까지 사람의 제스 쳐를 세부적인 목적/의도를 기준으로 구분하였다[2]. 본 연 
구를 통해 관찰한 $\mathrm{TV}$ 조작의 과정에서는 주어진 명령을 수 행하는 의도를 이미 가지고 있었기 때문에 제스티큘레이션 은 관찰 대상에서 제외하였고 Buxton(2011) 및 Karam (2005), Kendon(1988) 의 구분을 참조하여 Table 7 과 같 이 유형화 하였다.

Table 7. Types of gestures as intention of behavior

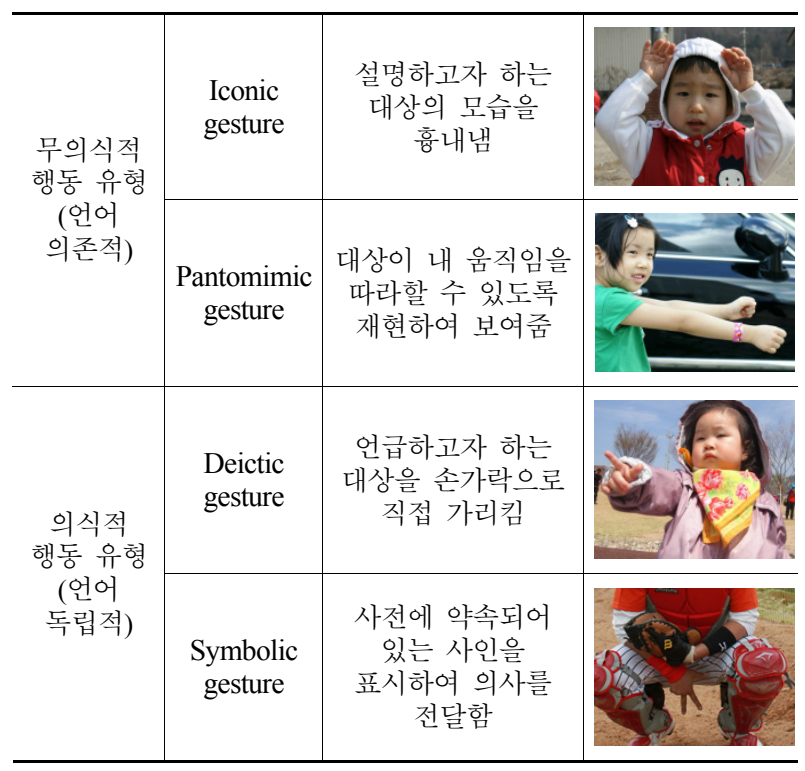

사물흉내(iconic gesture)의 경우, 사전에 합의된 바가 없이도 보편적으로 화자가 하는 말을 효과적으로 뒷받침해 주는 역할을 하나 사물을 흉내 내는 행동은 화자에 따라서 매번 달라진다. 상징 표현(symbolic gesture)의 경우에는 말을 하지 않고서도 의사를 전달할 수 있지만, 특정 기능에 일대일로 연결된 상징 (symbol)을 사인(sign)으로 사용하는 경우, 사전에 학습 및 상호간 합의가 반드시 필요하다. 그리 고 행동을 재현(pantomimic gesture)하거나 대상을 가리키 는(deictic gesture) 경우에는, 사전 학습이 없이도 직관적 으로 커뮤니케이션이 가능하다는 장점이 있다.

\subsubsection{Types of gestures as behavior}

사용자 행태 기반 입력방식에 있어서 크게 버튼 방식, 제 스쳐 방식으로 나눠 볼 수 있었으며 그 안에서 총 5 가지 Interaction으로 분류하여 1 차 분석을 실시하였다. 그 결과 Interaction 속성 별로 버튼 Press/Touch 방식, Space Gesture, Surface Gesture, Point \& Click, Symbolic Gesture 순서로 큰 비중을 차지하였다. 이를 통해 기존 TV 에서 사용되었던 버튼 입력 방식으로는 사용자 경험을 만족 시킬 수 없고 Operation Command 별로 다른 입력 방식을
제공해주어야 한다는 것을 알 수 있었다.

각 Operation Command 별로 사용자에게 적절한 입력 방식을 찾기 위해 기 분류된 Interaction을 각 속성에 맞 춰 Press-like, Gesture-like 기준 선상에 배치해 놓고 Operation Command를 유사한 Interaction 경향을 가지 고 있는 순서로 재 배열 작업을 하였다. 그 결과 Operation Command는 크게 순간적인 일회성 Command, 연속적인 값을 갖는 Command, 컨텐츠 선택, 컨텐츠 생성 등의 4 가 지로 분류가 되었다. 순간적인 일회성 Command에 해당하 는 전원, 취소, 메뉴실행, 순간저장은 Press like한 경향성을 보이며 연속성 Command에 해당하는 스크롤, 채널, 줌, 지 정, 입력은 Gesture-like한 경향성을 보였다. 하지만 볼륨 은 연속적인 값을 갖는 Command이지만 Press-like한 경 향성을 보인 Command로 분류가 되었다.

명령어에 따른 선호경향(Table 8)을 보면 순간적인 일회 성 조작명령 (Instant manipulation)은 버튼 누름과 유사한 모션을 선호하고, 연속적인 값을 갖는 명령 (Consecutive manipulation) 은 공간적 제스쳐를 선호한다는 것이다. 즉 일 회성 조작 명령에 대해서 특정한 심볼을 공중이나 표면에 그 리는 식의 행동은 잘 나타나지 않았다. 즉, 어떤 대상의 조작 을 위한 명령을 수행 시 언어를 완전히 대체할 수 있는 수

Table 8. Trend of preference according to features of commands

\begin{tabular}{|c|c|c|}
\hline & Command & 4 Preference \\
\hline \multirow{6}{*}{$\begin{array}{c}\text { Instant } \\
\text { one time } \\
\text { manipulation }\end{array}$} & Power_on & \\
\hline & Power_off & \\
\hline & Cancel & \\
\hline & Menu & \\
\hline & Home & \\
\hline & Capture & \\
\hline \multirow{7}{*}{$\begin{array}{l}\text { Consecutive } \\
\text { manipulation }\end{array}$} & Volume_+ & \\
\hline & Volume_- & \\
\hline & Scroll & \\
\hline & Channel_+ & \\
\hline & Channel_- & \\
\hline & Zoom_+ & \\
\hline & Zoom_- & \\
\hline Select & Screen/Element & \\
\hline \multirow{2}{*}{ Input } & Diagram & \\
\hline & Text & \\
\hline
\end{tabular}


준의 행동(symbolic gesture)을 수반하는 경우는 드물게 나타난다는 점이다. 기기의 조작을 위한 제스쳐는 사용자의 의사 표현을 보조하는 정도의 역할일 뿐, 제스쳐 자체가 명 령어로서 활용될 정도의 특이성/명시성을 갖는 경우는 드물 었다. 뿐만 아니라 대상을 가리키거나 시각적 피드백에 의존 하고자 하는 경향(deictic gesture)도 여러 개 중 하나를 선 택해야 하는 경우를 제외하고는 흔히 발생하지 않음을 볼 수 있었다(Table 8).

명령어의 성질은 입력장치의 종류에 따른 인터랙션 방식 에 괄목할만한 차이를 유발하지는 않는 것으로 확인되었다. 모든 명령어의 경우에서 행동을 재현하려는 의도의 제스쳐 (Manipulative gesture) 가 가장 주되게 수반되어 나타났다. 하지만 순간적인 일회성 명령어 및 지정을 위한 명령어에서 대상을 가리키거나 상징을 표현하는 방식의 제스쳐가 다소 나타났다(Table 9).

Table 9. Behavior style according to commands and input devices

\begin{tabular}{|c|c|c|c|c|}
\hline \multirow{2}{*}{ Command } & \multicolumn{4}{|c|}{ 입력장치 별 행동 의도/목적 비율 } \\
\hline & $\begin{array}{l}\text { Bare } \\
\text { hand }\end{array}$ & $\begin{array}{l}\text { Pad } \\
\text { type }\end{array}$ & $\begin{array}{l}\text { Bar } \\
\text { type }\end{array}$ & $\begin{array}{l}\text { Stick } \\
\text { type }\end{array}$ \\
\hline \multicolumn{5}{|l|}{ Power_on } \\
\hline \multicolumn{5}{|l|}{ Power_off } \\
\hline \multicolumn{5}{|l|}{ Cancel } \\
\hline \multicolumn{5}{|l|}{ Menu } \\
\hline \multicolumn{5}{|l|}{ Home } \\
\hline \multicolumn{5}{|l|}{ Capture } \\
\hline \multicolumn{5}{|l|}{ Volume_+ } \\
\hline \multicolumn{5}{|l|}{ Volume_- } \\
\hline \multicolumn{5}{|l|}{ Scroll } \\
\hline \multicolumn{5}{|l|}{ Channel_+ } \\
\hline \multicolumn{5}{|l|}{ Channel_- } \\
\hline \multicolumn{5}{|l|}{ Zoom_+ } \\
\hline \multicolumn{5}{|l|}{ Zoom_- } \\
\hline \multicolumn{5}{|l|}{ Screen/Elemen } \\
\hline \multicolumn{5}{|l|}{ Diagram } \\
\hline \multicolumn{5}{|l|}{ Text } \\
\hline & & & & \\
\hline
\end{tabular}

\subsubsection{Types of gestures as shapes of input devices}

입력 기기에 따른 제스쳐의 차이는 기기를 잡는 방식, 활 용 가능한 면의 면적, 기존 사용 경험 등의 영향을 받게 된
다. 따라서 $\mathrm{Pad} / \mathrm{Bar}$ type에서는 버튼 누르기나 표면 움직 임과 같이 형태의 특성을 반영한 행동들이 나타난다. 이 특 성들은 주로 사용자의 과거 경험의 영향으로 나타나지만, 행 동 의도(Intention)면의 분류에 기반해서 보면 근본적으로 대상물에 대해 흔히 나타날 수 있는 조작 행동을 재현하려는 판토마임적(Pantomimic)인 제스쳐로 볼 수 있다.

맨손의 경우, 공간에서의 '행동 재현'을 통해 연속적인 값 을 갖는 명령들, '화면요소선택', 그리고 '글자/도형의 입력' 면에 강점이 있다. 특정 입력 기기가 존재할 때에는 '버튼 누르기'의 선호 성향이 항상 존재하는데, 패드형에서는 '홈 으로', 바 형에서는 '글자 입력'이 상대적으로 강세를 보이는 것으로 미루어 기존의 타블렛PC 및 QWERTY 디바이스 사용 경험이 반영된 결과라고 볼 수 있다. 스틱형에서는 버 튼을 이용한 '볼륨 조절'이 적합한 인터랙션 기능으로 나타 났다.

Table 10. Optimal interaction method according to input devices

\begin{tabular}{|c|c|c|c|}
\hline Types & Behavior & $\begin{array}{l}\text { Intention / } \\
\text { Objective }\end{array}$ & Function \\
\hline $\begin{array}{l}\text { Bare } \\
\text { hand }\end{array}$ & $\begin{array}{l}\text { 공간 움직임 } \\
\text { (Space gesture) }\end{array}$ & $\begin{array}{c}\text { 행동재현 } \\
\text { (Pantomimic } \\
\text { gesture) }\end{array}$ & $\begin{array}{c}\text { Volume } \\
\text { Scroll } \\
\text { Channel } \\
\text { Zoom } \\
\text { Screen / Select } \\
\text { Input }\end{array}$ \\
\hline \multirow{2}{*}{$\begin{array}{l}\text { Pad } \\
\text { type }\end{array}$} & $\begin{array}{c}\text { 버튼 누르기 } \\
\text { (Button } \\
\text { Press/Touch) }\end{array}$ & \multirow{2}{*}{$\begin{array}{c}\text { 행동재현 } \\
\text { (Pantomimic } \\
\text { gesture) }\end{array}$} & $\begin{array}{l}\text { Power } \\
\text { Home }\end{array}$ \\
\hline & $\begin{array}{c}\text { 표면 움직임 } \\
\text { (Surface gesture) }\end{array}$ & & $\begin{array}{l}\text { Scroll } \\
\text { Zoom } \\
\text { Input }\end{array}$ \\
\hline $\begin{array}{l}\text { Bar } \\
\text { type }\end{array}$ & $\begin{array}{c}\text { 버튼 누르기 } \\
\text { (Button } \\
\text { Press/Touch) }\end{array}$ & $\begin{array}{c}\text { 행동재현 } \\
\text { (Pantomimic } \\
\text { gesture) }\end{array}$ & $\begin{array}{l}\text { Power } \\
\text { Input }\end{array}$ \\
\hline \multirow{2}{*}{$\begin{array}{l}\text { Stick } \\
\text { type }\end{array}$} & $\begin{array}{c}\text { 버튼 누르기 } \\
\text { (Button } \\
\text { Press/Touch) }\end{array}$ & \multirow{2}{*}{$\begin{array}{c}\text { 행동재현 } \\
\text { (Pantomimic } \\
\text { gesture) }\end{array}$} & $\begin{array}{l}\text { Capture } \\
\text { Volume }\end{array}$ \\
\hline & $\begin{array}{l}\text { 공간 움직임 } \\
\text { (Space gesture) }\end{array}$ & & $\begin{array}{c}\text { Scroll } \\
\text { Channel } \\
\text { Screen / Select }\end{array}$ \\
\hline
\end{tabular}

또한 패드형에서는 표면 위에서의 핀치(pinch), 플릭 (flick) 등이, 스틱형에서는 조작 대상을 입력 기기 끝으로 가리켜 원하는 방향 $(\mathrm{X}, \mathrm{Y}, \mathrm{Z}$ 방향) 으로 움직이는 판토마임적 행동이 주로 나타나 '스크롤', '줌' 등의 기능에 대응하는 것이 적합함을 알 수 있었다(Table 9).

본 연구의 또 다른 발견점 중 하나는, 스틱형 입력 기기를 
잡고 명령을 수행할 때와 맨손으로 수행할 때 매우 유사한 형태의 인지 모델을 바탕으로 한다는 점이다. 예를 들면, '스 크롤'을 위해 가상의 대상물을 손바닥이 향한 방향으로 밀어 내거나 '확대'를 위해 얼굴 가까이 대상을 끌어오는 방식이 두 경우에서 동일하게 차용되고 있다. '채널'의 경우 좌우 방 향으로 연속되게 나열된 이미지를 플리킹 (flicking)하여 불 러내는 방식의 행동을 취하는 것이었다(Table 11).

Table 11. A comparison of behavior between using stick type and bare-hand

\begin{tabular}{|c|c|c|c|}
\hline & Stick & Bare hand & Similarity \\
\hline Scroll & & & $\begin{array}{c}\text { 가상의 물체를 } \\
\text { 손으로 밀거나 } \\
\text { 잡아서 움직이 } \\
\text { 는 동작 }\end{array}$ \\
\hline $\begin{array}{l}\text { Channel } \\
\text { Zapping }\end{array}$ & & & $\begin{array}{l}\text { 좌우 방향으로 } \\
\text { 연속되게 나열 } \\
\text { 된 이미지를 불 } \\
\text { 러내는 동작 }\end{array}$ \\
\hline Zoom & & & $\begin{array}{c}\text { 가까이 끌어당 } \\
\text { 기거나 멀리 밀 } \\
\text { 어내는 동작 }\end{array}$ \\
\hline $\begin{array}{l}\text { Screen / } \\
\text { Element } \\
\text { Selection }\end{array}$ & & & $\begin{array}{c}\text { 여러 개 중 } \\
\text { 하나를 가리키 } \\
\text { 는 동작 }\end{array}$ \\
\hline $\begin{array}{l}\text { Text / } \\
\text { Diagram } \\
\text { Input }\end{array}$ & & & $\begin{array}{c}\text { 글자나 도형을 } \\
\text { 허공에 그려내 } \\
\text { 는 동작 }\end{array}$ \\
\hline
\end{tabular}

\subsection{Discussion}

스마트홈 환경에서 원거리의 대상에 대하여 통합적이고 직관적인 조작을 위한 최적의 대안으로서 맨손 제스쳐가 주 목 받고 있다 $[1,5]$. 사용자 중심의 자연적 인터랙션방식의 탐색 결과, 스틱형 입력장치를 이용한 '행동 재현적 공간 움 직임'은 맨손 상태에서의 행동을 가장 유사하게 반영하는 입 력 방식임을 확인할 수 있었다. 이러한 발견점을 바탕으로 맨손 상태 및 스틱형 입력장치를 이용하는 경우의 자연적 인 터랙션 행동을 면밀히 비교하여 정교화한다면, 향후 맨손 인 터랙션 환경에 대비한 과도기적 대안으로서 스틱형 제스쳐 입력 기기를 제안할 수 있을 것이다.

입력장치의 종류에 따라서 조작 행동의 형태는 확연한 차
이를 보였다(Figure 1). '공간 움직임'과 '표면 움직임'은 상호 정 반대의 증감 추이를 보이는데, 물리적인 표면이 넓어질수 록 버튼에의 의존도는 낮아지고, 직접적인 움직임을 통해 인 터랙션 하고자 함을 관찰할 수 있었다. '스틱형의 입력장치'에 서는 '공간 움직임'만큼 중요한 비중의 인터랙션 방식이 '버 튼 누르기'임을 알 수 있었다.

명령어의 성격은, 모든 입력장치의 경우에서 강력한 변인 으로 작용함을 관찰할 수 있었다. '전원', '취소', '메뉴실행', '순 간저장 등과 같이 순간적으로 입력하고 즉각적인 반응을 기 대하는 명령어의 경우는 버튼을 선호하는 경향이, '스크롤', '채널', '줌' 등과 같이 사용자의 연속적인 조작이 연속적인 피 드백으로 주어지도록 기대되는 명령어의 경우에는 제스쳐를 선호하는 경향이 현저히 강하게 나타났다.

특이한 점은, '볼륨'의 경우에는 연속적인 값을 가짐에도 불 구하고 연속적 피드백이 가능한 제스쳐 방식 보다 단계적인 증감을 유발하는 버튼 방식이 선호된다는 것이다.

\section{Conclusion}

본 연구는 향후 보급이 확산될 것으로 예상되는 Smart $\mathrm{TV}$ 를 대상으로 사용자의 멘탈 모델과 기기의 Core control scene 사이에서 벌어지는 최적의 인터페이스를 실험을 통하 여 검증함으로써 사용자에게 보다 쉽고 편리한 UX를 제공 하기 위하여 진행되었다. 그 결과 사용자의 멘탈 모델도 다 양한 제품의 사용 경험에 따라 변화한다는 것을 확인할 수 있었고, 기기의 Core control scene을 조작할 때도 입력장 치의 형상보다는 기능 속성에 따라 사용자의 행동/행태가 달 라지는 것을 확인 할 수 있었다.

Smart TV는 타 기기와 연결되고 넘쳐나는 서비스, 컨텐 츠를 소비하는 제품이다. 이번 연구에서는 이런 복잡한 사용 환경 속에서 인간 중심의 솔루션 제안으로 개발에 바로 적용 가능한 실용적 연구성과를 도출할 수 있었다. 과거 기업에서 $\mathrm{UX}$ 개발 시, 신기술 위주로 UI를 구성하였다면 이번 접근법 은 사용자의 본질적 행동 행태를 관찰하고 분석한 결과를 개 발에 적용할 수 있는 토대를 마련한 것이다.

비록 이번 연구는 Smart TV의 입력장치 위주의 사용 경 험을 중심으로 연구되었으나 향후에는 이런 방법론을 다양 한 가전 제품군에 확대 적용한다면 인간 중심의 제품과 서 비스 경험을 개발하여 경쟁력 있는 상품으로 연결할 수 있 을 것이다. 


\section{References}

Buxton, B., Chap 14: Gesture based interaction. Haptic input. Publishing. 2011. http://www.billbuxton.com/input14.Gesture.pdf

Holman, D., Vertegaal, R. \& Altosaar, M. PaperWindows: Interaction Techniques for Digital Paper. Proc. CHI 2005, ACM Press, 591-599, 2005.

Kendon, A., Sign Languages of Aboriginal Australia: Cultural, Semiotic and Communicative Perspectives. Cambridge: Cambridge University Press, Pp. xviii+ 542, 1988.

Lee, S., "How users manipulate deformable displays as input devices", In Proc. of CHI '10, ACM, 1647-1656, 2010.

Karam, M., "A taxonomy of Gesture in Human Computer Interaction", Technical Report ECSTR-IAM05-009, Electronics and Computer Science, University of Southampton, 2005.

Neßelrath, R., Lu, C., Schulz, H. C., Frey, J. \& Alexandersson, J., A Gesture Based System for Context-Sensitive Interaction with Smart Homes. Ambient Assisted Living: 4. AAL-Kongress 2011 Berlin, Germany. p. 209-219, 2011.

Cho, S. Hong, S, Hong, J. Jang J. \& Choi, J., Study of smart TV input method according to operation command. Korean HCI 2012. Proceeding.

Wobbrock, J. O., Morris, M. R. and Wilson, A. D., "User-defined gestures for surface computing", Proc. CHI 2009, ACM Press, 1083-1092, 2009.

\section{Author listings}

Jinhae Choi: jin.choi@lge.co.kr

Highest degree: $\mathrm{PhD}$, Human Environment Design and Science, Chiba University

Position title: Principal Researcher, UX Lab, LG Electronics Inc.

Areas of interest: UX Design, Human Centered Design, HCI

Jiyoung Hong: jiyoung.hong@lge.co.kr

Highest degree: $\mathrm{PhD}$, Cognitive Science, Yonsei University Position title: Chief Researcher, UX Lab, LG Electronics Inc.

Areas of interest: UX Design, Cognitive Engineering, HCI

Date Received : 2012-07-29

Date Revised : 2012-08-07

Date Accepted : 2012-08-07 